\title{
FIRST MEASUREMENT OF THE VECTOR ANALYZING POWER IN MUON CAPTURE BY POLARIZED MUONIC ${ }^{3}$ HE
}

W.J. Cummings ${ }^{3 *}$, J. Behr ${ }^{1}$, P. Bogorad ${ }^{4}$, E.J. Brash ${ }^{9}$, G.D. Cates ${ }^{4}$, A. Gorelov ${ }^{1}$, M.D. Hasinoff ${ }^{2}$, O. Hausser ${ }^{1,3}$, K. Hicks ${ }^{7}$, R. Holmes 5 , J.C. Huang ${ }^{2}$, K. Kumar ${ }^{4}$, B. Larson ${ }^{7}$, W. Lorenzon ${ }^{8}$, J. McCracken ${ }^{5}$, P. Michaux ${ }^{3}$, H. Middleton ${ }^{4}$, E. Saettler ${ }^{2}$, D. Siegel ${ }^{4}$, P.A. Souder ${ }^{5}$, D.

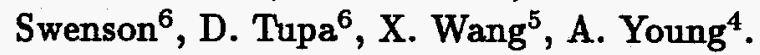

${ }^{1}$ Simon Fraser University, Burnaby, B.C., Canada V5A 156

${ }^{2}$ University of British Columbia, Vancouver, B.C., Canada V6T $1 Z 1$

${ }^{3}$ TRIUMF, Vancouver, B.C., Canada V6T $2 A 3$

${ }^{4}$ Princeton University, Princeton, NJ USA

${ }^{5}$ Syracuse University, Syracuse, NY USA

${ }^{6}$ Los Alamos National Lab, Los Alamos, NM USA

${ }^{7}$ Ohio University, Athens, $O H 45701$ USA

${ }^{8}$ University of Pennsylvania, Philadelphia, PA USA

${ }^{9}$ Rutgers University, Piscataway, NJ USA

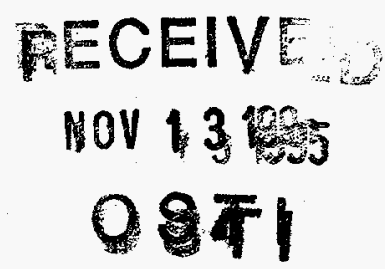

\section{ABSTRACT}

This paper describes the first measurement of spin observables in nuclear muon capture by ${ }^{3} \mathrm{He}$. The sensitivity of spin observables to the pseudoscalar coupling is described.

\section{Introduction}

One goal of studying nuclear muon capture is to use the well understood theory of the electro-weak interactions to probe the form factors which describe the hadronic vertex. In a semileptonic weak process such as nuclear muon capture the purely weak (vector - axial vector) current is modified by the addition of four induced form factors. Of these additional couplings (weak magnetic, scalar, pseudoscalar and tensor), the scalar and tensor terms are known to be very small. ${ }^{1}$ The remaining four form factors can be related to other electroweak measurements using the conserved vector current (CVC) theorem and the partially conserved axial current (PCAC) hypothesis. The CVC theorem allows values for the vector and weak magnetic form factors to be extracted from electron scattering data. The axial current $g_{A}$ can be extrapolated to the muon capture $q^{2}$ from beta decay measurements at $q^{2}=0$. The induced pseudoscalar $g_{P}$ term is proportional to $q^{2}$ and therefore is not studied in beta decay. However, the ratio of $g_{P} / g_{A}$ can be predicted using the PCAC hypothesis and the Goldberger-Treiman relation. This prediction can only be tested using muon capture data. For the fundamental reaction of ordinary muon capture (OMC) by hydrogen, this prediction ${ }^{2}$ is $g_{P}\left(q^{2}=-0.88 m_{\mu}^{2}\right)=6.77 g_{A}\left(q^{2}=0\right)$. Experimentally, the measurement of $g_{P}$ from the ordinary muon capture (OMC) rate in hydrogen is very difficult because of

\footnotetext{
"Present Address:

Physics Division, Argonne National Laboratory, Argonne II 60439; E-mail: cummings@anl.gov
} 
the weak sensitivity of the total capture rate to the pseudoscalar part. The best single measurement $(4 \%)$ of the OMC rate only gives a $40 \%$ measurement of $g_{P} .^{3}$ The world average of all $\mathrm{OMC}$ measurements gives the result $g_{P} / g_{A}=6.9 \pm 1.5$ in agreement with the PCAC estimate. ${ }^{3}$

Recently, the importance of a more precise measurement of $g_{P}$ was reinforced by a new $3 \%$ theoretical prediction using QCD chiral Ward identities. ${ }^{4} \mathrm{~A}$ more precise determination of $g_{P}$ was the motivation of the radiative muon capture (RMC) on hydrogen experiment at TRIUMF. In RMC the momentum transfer can be varied, with an enhanced sensitivity to $g_{P}$ (by roughly a factor of three) occurring near the pole of the pion propagator. In spite of the unfavorable $\mathrm{RMC} / \mathrm{OMC}$ ratio of $10^{-5}$, this group has released a $12 \%$ measurement of $g_{P}$ at this conference. However, their result is 2.5 standard deviations above the PCAC prediction. ${ }^{5}$ This suprising result shows a clear need for additional measurements of the pseudoscalar term with similar accuracy.

A comparison of $g_{P}$ from muon capture in nuclei with $g_{P}$ from muon capture in hydrogen is of interest since one-pion exchange dominates the pseudoscalar part of the semileptonic current. Meson exchange currents (MEC) in nuclei may modify $g_{P}$ from the hydrogen/PCAC value. Of all nuclear targets, ${ }^{3} \mathrm{He}$ is the most interesting for muon capture studies. Muon capture on ${ }^{3} \mathrm{He}$ has the advantage that the ${ }^{3} \mathrm{He} \rightarrow{ }^{3} \mathrm{H}$ transition has the same spin, isospin and parity as the $p \rightarrow n$ transition. The wavefunctions of the $A=3$ isodoublet can be calculated with little uncertainty from Faddeev methods. In addition, tritons emitted following muon capture by polarized muonic ${ }^{3} \mathrm{He}$ exhibit a characteristic asymmetry which allows a nearly model independent extraction of $g_{P}$ with enhanced sensitivity over capture rate measurements. TRIUMF Experiment 683 has recently completed the first measurement of this asymmetry.

\section{Theory}

The observation of spin dependence in muon capture from ${ }^{3} \mathrm{He}$ implies an increase in sensitivity similar to that between RMC and OMC. Congleton and Fearing ${ }^{6}$ have calculated the spin dependence of the reaction $\mu^{-}+{ }^{3} \mathrm{He} \rightarrow t+\nu_{\mu}$ by treating the $\mathrm{A}=3$ isodoublet as "elementary particles". They show that the spin dependence of the triton rate (the triton branch is $0.3 \%$ ) can be expressed as: ${ }^{a}$

$$
\frac{d \Gamma}{d \Omega}=\frac{\Gamma_{o}}{4 \pi}\left[1+P_{v} A_{v} \cos \theta\right]
$$

where $\Gamma_{o}$ is the unpolarized rate, $\theta$ is the angle between the polarization axis and the triton direction, and $\boldsymbol{P}_{v}$ is the vector polarization of the muonic ${ }^{3} \mathrm{He}$ atom. They have calculated the dependence of the vector analyzing power $\left(A_{v}\right)$ and the total capture rate $\left(\Gamma_{o}\right)$ on the vector $\left(F_{v}\right)$, magnetic $\left(F_{m}\right)$, axial vector $\left(F_{a}\right)$ and pseudoscalar $\left(F_{p}\right)$ nuclear form factors. Using measured values for the first three form factors and the PCAC prediction for $F_{p}$, they obtain $\Gamma_{o}=1497 \pm 11 \mathrm{sec}^{-1}$ and $A_{v}=0.5243 \pm 0.0057$. With these values, the

${ }^{a}$ Neglecting terms from tensor polarization 


\section{DISCLAIMER}

Portions of this document may be illegible in electronic image products. Images are produced from the best available original document. 
predicted sensitivities to the value of $F_{p}$ have been calculated to be $\frac{\Delta \Gamma_{0}}{\Gamma_{0}}=0.11 \frac{\Delta F_{p}}{F_{p}}$ and $\frac{\Delta A_{v}}{A_{v}}=0.38 \frac{\Delta F_{p}}{F_{p}}$. One can easily see the advantage of measuring the spin dependent parts of the rate in making a measurement of $F_{p}$.

\section{Experiment}

The experiment requires that the muon beam is stopped, the muonic ${ }^{3} \mathrm{He}$ is polarized, and the triton is detected in the same gas volume. The experiment was performed at TRIUMF using very low momentum (21 MeV/c) muons from the M9B superconducting muon channel. These muons stop in a region containing high pressure ${ }^{3} \mathrm{He}$ gas and form muonic He atoms. The muonic ${ }^{3} \mathrm{He}$ atoms are polarized by spin exchange collisions with the optically pumped Rb vapor. Previous experiments at LAMPF have shown that the spin exchange cross sections are large enough to achieve large muonic ${ }^{3} \mathrm{He}$ polarization's within the muon lifetime. ${ }^{7}$ The $\mathrm{Rb}$ number density used was about $4 \times 10^{14}$ atoms $/ \mathrm{cm}^{3}$. The optical pumping light is provided by two diode laser arrays ${ }^{8}$ operating at the Rb D1 resonance $(795 \mathrm{~nm}$ ) which combined to give a total of $27 \mathrm{~W}$ of circularly polarized photons. The entire target apparatus is contained in an oven and operated at a temperature of $200 \mathrm{C}$ to maintain the required $R b$ vapor density.

The muonic ${ }^{3} \mathrm{He}$ vector polarization is monitored using the decay electron asymmetry from muons which decay after the muonic He atom has been polarized. These decay electrons are detected by a detector telescope, consisting of two wire chambers to measure the direction of the detected electron and a scintillator absorber stack to record only the highest energy electrons (which have the largest analyzing power). The experimental asymmetry is created by comparing the the rate of decay electrons with respect to the circular polarization of the optical pumping photons (which was reversed every 200 seconds). The electron events are analyzed to determine the origin of the electron. These reconstructed decay electron events are compared with the results of a complete GEANT Monte Carlo simulation. This comparison is used to deduce the muon vector polarization from the decay electron asymmetry.

The tritons created by nuclear muon capture are monoenergetic with $1.9 \mathrm{MeV}$ of kinetic energy and therefore stop within the gas volume of the target. They are detected by an ionization chamber contained within the target. This detector detects both the ionization of the incoming muon and the recoil triton. A drift field causes this ionization to drift in the same direction as the optical pumping laser beam until it is collected at the anode. The ionization collected at the anode is split into two signals: an inner active circular disc which is the same size as the incoming laser beam and an outer veto ring used to reject events which come from outside the region of the laser beam and are therefore unpolarized. Both signals are recorded using a $10 \mathrm{MHz}$ flash adc (FADC) which records the entire drift time of the ionization chamber $(100 \mu \mathrm{sec})$. The time evolution of the ionization arriving at the anode is used to determine the direction of the recoil triton $(\cos \theta)$.

For muon capture events, the ionization chamber information is used to reconstruct 

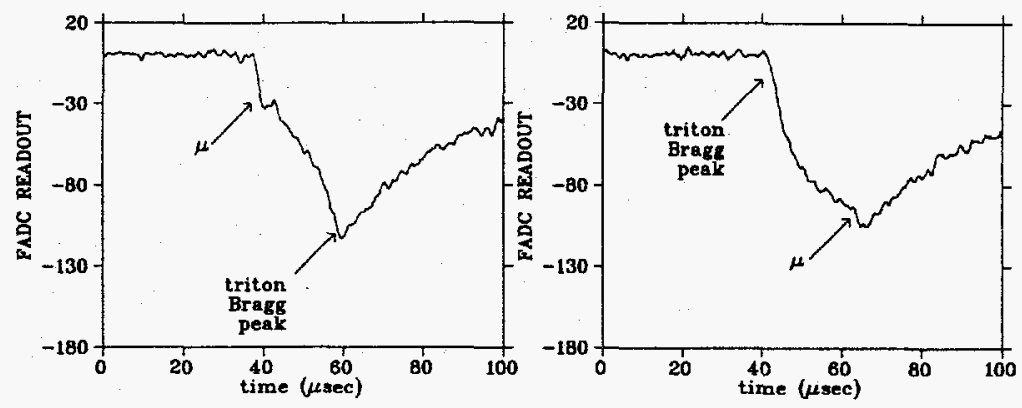

Figure 1: FADC outputs from two E683 triton events showing the ionization chamber signals.

both the muon and triton tracks and determine the direction of the emitted triton. In general, interesting muon capture events fall into two categories. In the first type of event, the triton is emitted and travels against the drift field. These events which travel upstream, we call "uppers." The other type of interesting event occurs when the triton is emitted in the same direction as the drift field. These downstream events are labelled "downers." These events are interesting because they have the large values of $\cos \theta$ needed to measure $A_{v}$.

For each muon capture event, the FADC information is analyzed to determine if the event is interesting. To explain how this analysis works, two interesting events are shown in Fig. 1. The left side shows the inner anode FADC data from a typical "upper" pulse For this type of event, the ionization from the muon track arrives at the anode first. Just after the muon, the triton ionization arrives at the inner anode. The rate of ionization arriving at the anode is measured by the slope of rising triton pulse. The most intense ionization on the triton track occurs at the Bragg peak where the triton stops. This intense ionization is visible as the steep slope just before the peak of the pulse. The remainder of the pulse falls with the characteristic RC time constant of the amplifier circuit.

By contrast, the left side of Fig. 1 show the inner anode FADC data from a typical "downer" pulse. The very different pulse shape of this type of event is caused by the fact the ionization from the triton Bragg peak arrives first at the anode. The ionization from the muon arrives later as the small bump seen on top the triton ionization.

As illustrated by these two examples, these two types of events can easily be distinguished from one another by the curvature of the triton pulse risetime and the location of the muon ionization. In a more detailed analysis, the pulse shape of every interesting event is fit to determine the $\cos (\theta)$ of the detected triton. The triton rates can then be compared for the two different helicities of the optical pumping laser light to determine the triton asymmetry.

\section{Preliminary Results}

Our preliminary results for the triton asymmetry are found in Fig. 2. This asymmetry 


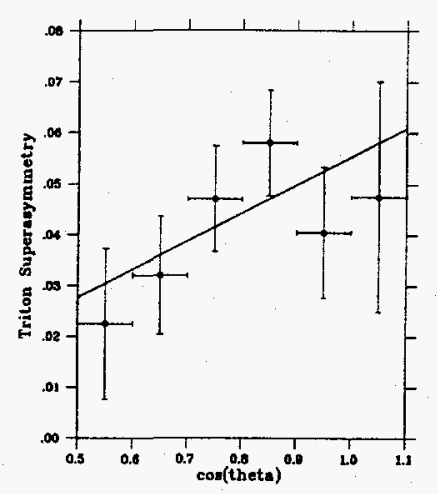

Figure 2: Triton asymmetry shown as a function of $\cos (\theta)$.

is calculated using a "superasymmetry" formula:

$$
A=\frac{\sqrt{N_{C C W}^{\text {up }} N_{C W}^{\text {down }}}-\sqrt{N_{C C W}^{\text {down }} N_{C W}^{\text {up }}}}{\sqrt{N_{C C W}^{\text {up }} N_{C W}^{\text {down }}}+\sqrt{N_{C C W}^{\text {down }} N_{C W}^{\text {up }}}},
$$

where the subscripts $\mathrm{CCW}$ and $\mathrm{CW}$ refer to optical pumping light helicities. These results are shown as a function of $\cos (\theta)$ for 6 bins between 0.5 and 1.1. Below $\cos (\theta)=0.5$, our acceptance for triton events was very small. Above $\cos (\theta)=1.0$, there are still triton events because of the angular resolution of the ionization chamber. The straight line shown on Fig. 2 is a fit to the expected shape of this distribution from Eq. 1. The agreement with this distribution is very good.

\section{Conclusions}

The triton asymmetry presented in the last section has to be corrected for small systematic effects in order to extract the vector analyzing power. The analysis of these effects is currently underway. However, our first measurement has already validated a powerful new method for studying the weak interaction. Our collaboration plans a new measurement with more powerful laser beams and an improved ionization chamber. The ultimate goal is a measurement of $F_{P}$ of $10 \%$ accuracy to compare to the RMC result.

This work is supported by NSERC,NRC (Canada) and DOE (USA) grants.

1. P. Baringer et al., Phys. Rev. Lett. $\underline{59}, 1993$ (1987).

2. H.W. Fearing, Phys. Rev. C21, 1951 (1980).

3. G. Bardin et al., Nucl. Phys. A352, 365 (1981).

4. V. Bernard, N. Kaiser, Ulf-G. Meissner, Phys. Rev. D50, 6899 (1994).

5. G. Jonkmans et al., to be published; and M. Blecker, this conference.

6. J.G. Congleton and H.W. Fearing, Nucl. Phys. A552, 534 (1993).

7. A. Barton et al., Phys. Rev. Lett.70, 758 (1993).

8. W.J. Cummings et al., Phys. Rev. A51, 4842 (1995). 


\section{DISCLAIMER}

This report was prepared as an account of work sponsored by an agency of the United States Government. Neither the United States Government nor any agency thereof, nor any of their employees, makes any warranty, express or implied, or assumes any legal liability or responsibility for the accuracy, completeness, or usefulness of any information, apparatus, product, or process disclosed, or represents that its use would not infringe privately owned rights. Reference herein to any specific commercial product, process, or service by trade name, trademark, manufacturer, or otherwise does not necessarily constitute or imply its endorsement, recommendation, or favoring by the United States Government or any agency thereof. The views and opinions of authors expressed herein do not necessarily state or reflect those of the United States Government or any agency thereof. 